\title{
Percutaneous intracardiac beating-heart surgery using metal MEMS tissue approximation tools
}

\section{Citation}

Gosline, Andrew H, Nikolay V Vasilyev, Evan J Butler, Chris Folk, Adam Cohen, Rich Chen, Nora Lang, Pedro J del Nido, and Pierre E Dupont. 2012. "Percutaneous Intracardiac Beating-Heart Surgery Using Metal MEMS Tissue Approximation Tools." The International Journal of Robotics Research 31 (9) (May 8): 1081-1093. doi:10.1177/0278364912443718.

\section{Published Version}

doi:10.1177/0278364912443718

\section{Permanent link}

http://nrs.harvard.edu/urn-3:HUL.InstRepos:33884744

\section{Terms of Use}

This article was downloaded from Harvard University's DASH repository, and is made available under the terms and conditions applicable to Other Posted Material, as set forth at http:// nrs.harvard.edu/urn-3:HUL.InstRepos:dash.current.terms-of-use\#LAA

\section{Share Your Story}

The Harvard community has made this article openly available.

Please share how this access benefits you. Submit a story.

\section{Accessibility}




\title{
Percutaneous intracardiac beating-heart surgery using metal MEMS tissue approximation tools
}

\author{
Andrew H Gosline ${ }^{1}$, Nikolay V Vasilyev ${ }^{1}$, Evan J Butler ${ }^{1}$, Chris Folk ${ }^{2}$, Adam Cohen ${ }^{2}$, Rich \\ Chen $^{2}$, Nora Lang ${ }^{1}$, Pedro J del Nido ${ }^{1}$, and Pierre E Dupont ${ }^{1}$ \\ ${ }^{1}$ Cardiovascular Surgery, Children's Hospital Boston, Harvard Medical School, Boston, MA, USA \\ ${ }^{2}$ Microfabrica, Inc., Van Nuys, CA, USA
}

\begin{abstract}
Achieving superior outcomes through the use of robots in medical applications requires an integrated approach to the design of the robot, tooling and the procedure itself. In this paper, this approach is applied to develop a robotic technique for closing abnormal communication between the atria of the heart. The goal is to achieve the efficacy of surgical closure as performed on a stopped, open heart with the reduced risk and trauma of a beating-heart catheter-based procedure. In the proposed approach, a concentric tube robot is used to percutaneously access the right atrium and deploy a tissue approximation device. The device is constructed using a metal microelectromechanical system (MEMS) fabrication process and is designed to both fit the manipulation capabilities of the robot as well as to reproduce the beneficial features of surgical closure by suture. The effectiveness of the approach is demonstrated through ex vivo and in vivo experiments.
\end{abstract}

\section{Keywords}

image guided surgery; robotic surgery; MEMS tools; concentric tube robots; intracardiac surgery

\section{Introduction}

Existing surgical robots tend to mimic the techniques and tools used by surgeons in manual surgery. For example, robotic tools for minimally invasive surgery are often identical to the manual tools used to perform laparoscopic procedures, e.g. forceps, scissors, needles and sutures (Madhanir et al., 1998; Simaan et al., 2009). This approach can facilitate clinical adoption since it builds on the clinician's existing skill set.

There are many surgeries, however, where the robotization of current techniques is either not feasible or does not produce improvements in outcome or efficiency. For robots to be effective in these situations, new surgical techniques and tools are needed to fit the capabilities of robots.

One such application is intracardiac surgery. While the demand for heart surgery is increasing, open-heart procedures that require stopping the heart are declining due to inherent clinical risks, creating opportunities for devices that can perform intracardiac procedures in a minimally invasive fashion on the beating heart. While catheters provide

(c) The Author(s) 2012

Corresponding author: Andrew H Gosline, Children's Hospital Boston, 300 Longwood Avenue, Boston, MA 02115, USA. andrew.gosline@childrens.harvard.edu. 
these capabilities for certain procedures, their inherent flexibility substantially limits their tip forces and positioning accuracy and so constrains the tools and procedures they can perform.

As a specific example, consider the closure of patent foramen ovales (PFOs). Depicted in Figure 1(a), the foramen ovale is an opening between the two atria of the heart that allows blood to circulate while bypassing the lungs prior to birth. It should close spontaneously shortly after birth but fails to close completely (is patent) in about $27 \%$ of the population (Kizer and Devereux, 2005). A PFO can allow blood returning from the body to be shunted from the right to left atrium without first circulating to the lungs for filtration and oxygenation. Many individuals with a PFO show no symptoms in their lifetime, but clots or particles in the blood in the right atrium that cross a PFO into the left atrium can cause strokes or heart attacks (Kerut et al., 2001).

Surgical closure by suture has been considered the gold standard of treatment (Dearani et al., 1999), however, this approach is highly invasive and involves placing the patient on cardiopulmonary bypass and stopping the heart. Owing to the risks of open-heart surgery, this approach is currently rarely undertaken unless the patient is undergoing open-heart surgery for other reasons.

Figure 1(b) depicts a porcine PFO and illustrates that the PFO is a tunnel-like channel that runs almost parallel to and between the tissue layers of the septum secundum and septum primum. In more severe cases (not shown), the septum primum does not extend under the septum secundum creating a hole directly connecting the atria. To close either a channel or a hole, surgical technique, as shown in Figure 1(c), typically involves stretching the septum secundum to produce sufficient overlap with the septum primum (see the arrows in Figure 1(b)) and then placing a running suture that penetrates and approximates both layers.

Alternatively, PFOs can be closed using catheter-deployed occlusion devices inside the beating heart (Mareedu et al., 2007). These devices are inserted through the PFO itself and act as a plug against blood flow. One such device (Cardioseal, NMT Medical), as shown in Figure 2(a), is a double umbrella design that pops open when pushed out of a catheter. It is simple to deploy since the catheter need only pass through the existing PFO channel and extend an umbrella on each side of the hole. These simple motions are in contrast to those of closure by suture, which involves applying forces to overlap and pierce the tissue layers as well as dexterous manipulation of needle and suture.

Closure using catheter-delivered occlusion devices is achieved in more than $90 \%$ of the cases (Khairy et al., 2003; Kizer and Devereux, 2005); however, this approach has several disadvantages. First, as shown in Figure 2(c), a significant amount of foreign material is placed inside the left atrium leading to the possibility of thrombus formation and embolization (Khairy et al., 2003). Second, unlike closure by suture, the devices are not adjustable, but rather are held in place by the elastic relaxation of their Nickel Titanium (NiTi or nitinol) structural elements. While this approach facilitates deployment, it does not provide the patient-specific adjustability of surgical closure potentially leading to insufficient or to excessive approximation forces.

The ideal PFO closure device would be delivered percutaneously, like catheter-delivered devices, but also provide adjustability, reliability and introduce the minimum possible amount of foreign material, as is obtained in suture closure. There are several challenges to satisfying these requirements. First, there is very little room in the right atrium to perform complex manipulations. Second, despite recent advances in robotic catheter systems (Meeker et al., 1996; Camarillo et al., 2008, 2009; Ikeuchi and Ikuta, 2009; Jayender et al., 2009), the stiffness necessary to displace the septum secundum and the dexterity and positioning accuracy necessary to mimic suturing appear incompatible to catheter delivery. 
The contribution of this paper is to propose a solution composed of a robotic delivery platform and a tissue approximation device designed to fit the capabilities of the robotic platform (Butler et al., 2011). The delivery platform, as illustrated in Figure 3, is a concentric tube robot that is used for percutaneous access to the right atrium via the internal jugular vein in the neck. Substantially stiffer than a catheter, the robot can apply higher forces to manipulate tissue inside the heart. Concentric tube robots are a novel class of continuum robots composed of nested precurved elastic tubes that are well suited to minimally invasive surgery (Dupont et al., 2010; Lock et al., 2010; Rucker et al., 2010a, 2010b; Anor et al., 2011; Bedell et al., 2011; Lock and Dupont, 2011; Mahvash and Dupont, 2011).

The tissue approximation device, Figure 2(b,d), takes advantage of the robot's force capabilities to adjustably approximate the two tissue layers (septum primum and septum secundum of Figure 1(a)) in a manner comparable to suturing while not requiring the complex manipulations involved in throwing stitches and tying knots. Since the portion of the device protruding from the tissue is comparable in size to the suture used in surgical closure (compare Figures 1(c) and 2(d)), it would be rapidly encapsulated in the tissue and would not carry the risks of the existing occlusion devices (Figure 2(c)).

The paper is arranged as follows. The next section describes the metal microelectromechanical system (MEMS) process used to manufacture the tissue approximation device. Section 3 details the design of the approximation device and the procedure to deploy it. Section 4 describes the design of the robotic system. Section 5 presents experimental results. These include ex vivo experiments, which were used to develop the detailed surgical procedure, as well as in vivo beating-heart experiments, which were used to validate the robotic closure system. A discussion of the results appears in the final section of the paper.

\section{Metal MEMS device fabrication}

Currently, millimeter-scale surgical instruments and implants are produced in metal by methods such as numerically controlled machining, electrical discharge machining, grinding, and laser machining (e.g. for stents). In some cases, welding and forming methods may also be used, and for some components (if not too small), metal injection molding may be suitable.

These methods suffer from one or more constraints. These include the inability to make extremely small features (e.g. due to material deflection, inability to fixture/hold the workpiece) and the difficulty in making internal features (due to restricted tool access). In addition, device components are manufactured serially by these methods and often must be subsequently manually assembled under a microscope, resulting in high manufacturing costs.

In contrast, the metal MEMS technology used here (Microfabrica, Inc.) is an additive, freeform metal manufacturing process for volume manufacturing of micrometer- and millimeter-scale devices. Based on a 3D CAD model of the desired device, the process stacks independently formed and patterned metal layers. The technology allows designers to create intricate 3D geometries with micrometer-level precision. It is a batch, wafer-scale process in which many devices are built simultaneously, allowing volume production at low cost.

As illustrated in Figure 4, the fabrication process utilizes a structural metal and a sacrificial metal. The structural metal is typically a nickel-cobalt alloy while the sacrificial metal is copper. Nickel cobalt has sufficient biocompatibility for use in surgical tools. For implants, 
however, long-term biocompatibility is required. Palladium (Pd) satisfies this requirement and is compatible with the fabrication process.

As a technology for millimeter-scale surgical devices, this process offers the following advantages. First, it permits the use of macro-scale mechanisms (e.g. hinges, slides, ratchets, chains, and pulleys) at the millimeter scale. This enables device designs with patient-topatient adjustability through the use of ratcheting or other mechanisms resulting in much finer control over the forces exerted on tissue. Second, it is economical since hundreds or thousands of pre-assembled devices can be manufactured in a single batch. Third, the fabrication process enables designs possessing superior material properties since it permits the use of combinations of structural metals, e.g. for high strength, hardness and biocompatibility.

\section{Device design}

The catheter motions to install occlusion devices, such as that shown in Figure 2(a), consist of steering the soft catheter tip through the PFO channel so that it enters the left atrium. The distal patch of the occlusion device is pushed from the end of catheter and its nitinol struts spring open so that the patch completely covers the septal channel. After pulling the patch against the septum, the proximal patch is similarly deployed on the proximal side in the right atrium and the device is released. The nitinol struts press both patches against the septum with a force that depends on the thickness of the patient's septal tissue.

In contrast to a catheter, a concentric tube robot possesses substantially higher longitudinal and bending stiffnesses as well as high precision control of the tip position. These qualities enable precise puncture and manipulation of the septal tissue and so provide more design freedom for a robot-delivered device. The right atrium is a confined space (several centimeters in diameter), however, providing little room for the robot to perform complex manipulations. Thus, the delivery procedure should require simple robot motions.

In the proposed procedure, instead of covering the hole with foreign material, the robot punctures the two tissue layers forming the channel (septum secundum and primum in Figure 1(a)) and the device, similar to suture closure, pulls them together just enough to seal the hole. The high axial and bending stiffnesses of the robot enable tissue puncture despite robot curvature. Furthermore, the robot's high bending stiffness enables it to replicate the surgical step of pulling the septum secundum laterally so that it overlaps the thicker tissue of the septum primum during approximation.

The device design specifications can be grouped as surgical requirements and robot delivery requirements.

Surgical requirements:

1. The distance that the tissue is approximated should be adjustable on a patientspecific basis.

2. Sufficient force should be applied between the approximating tissues to prevent their separation. Conservative estimates by the experienced cardiac surgeon coauthors of the tension applied during suture closure suggested a value of about 1 N.

3. The foreign material introduced into the heart should be as small as possible and not protrude into the left atrium.

Robot deployment requirements: 
4. The device must be compliant in bending so that it can be delivered through the curves of the concentric tube robot.

5. Device deployment must be reliable and allow for removal of the device middeployment.

An enlarged view of the final device is shown in Figure 5. Its cross section is $1.5 \mathrm{~mm}$ at its widest and it is $18 \mathrm{~mm}$ long. It has two pairs of expanding spring-loaded wings that are used to pull the tissue layers together. The distal wings attach to a toothed rail while the proximal wings are attached to a pawl flexure that engages the rail teeth forming a ratcheting mechanism, which adjusts the device length.

The delivery system consists of a tube and wire (not shown) at the proximal end of the device. The delivery wire screws into the device while the delivery tube mates with the device through a dog clutch.

Device deployment is illustrated schematically in Figure 6. During robotic deployment, a sharpened stylet inserted through the robot was used in place of the cannula. As depicted in Figure 6, the robot produces appropriate overlap of the tissue layers by first piercing the proximal layer and then dragging it laterally to achieve the desired overlap with the distal layer. The robot then punctures the second layer and proceeds with device deployment.

In the fourth deployment step shown in Figure 6, the deployment tube and wire are both extended to push the device out of the robot tip. Flexure springs cause the wings to rotate open by $30^{\circ}$ as they are extended from the robot to ensure that they will catch against the tissue. In the fifth step, relative translation of the deployment tube and wire causes the ratchet rail to shorten, pulling the tissue layers together while fully extending the wings. Finally, in step six, counterclockwise rotation of the deployment wire with respect to the deployment tube releases the device. Alternately, if the deployment is unsatisfactory, the distal wings can be released and the device removed by clockwise rotation.

In summary, the device employs three degrees of freedom during deployment. The first is rigid-body translation along the axis of the delivery device. The second degree of freedom is for the ratcheting to bring the tissue layers together. It is produced by relative translation of the deployment tube and wire. The third degree of freedom is relative rotation between the tube and wire to release the device.

Hundreds of the approximation devices are fabricated fully assembled on a single wafer using the metal MEMS process described in the preceding section. As shown in Figure 7, the device is composed of eight moving parts. Details of the design features and process are provided in the following sections.

\subsection{Device removal during deployment}

During deployment, it is important to be able to remove a device from the patient. Reasons include inadequate positioning of the device, malfunction, as well as changes in the condition of the patient. This feature is incorporated in the approximation device design.

To remove the device safely, it must be retracted into the robot without tearing any tissue that it has passed through and without any parts breaking off to be carried away by the blood stream. Functionally, this requires that the proximal and distal wings fold against the body of the device. The proximal wings will pivot in the correct direction during retraction. This is not true of the distal wings, however. 
The mechanism that enables both device release as well as removal is depicted conceptually in Figure 8. During normal operation, the rod shown in Figure 7 prevents the distal wings from folding outward. Once it is determined that the device is appropriately deployed, the device is released by rotating the threaded deployment wire counterclockwise while the deployment tube prevents the device from rotating.

If the device must be removed, however, the deployment wire is rotated clockwise with respect to the deployment tube. This causes rotation of the left-handed screw threads between the rod and rail of Figures 7 and 8 . The rod translates in the proximal direction with respect to the rail thus allowing the distal wings to fold outward and so be retracted through tissue without damage and into the robot.

\subsection{Compliance and strength}

The design specifications for bending compliance and pull-out force are challenging and conflicting requirements. The bending compliance requirement is for the device to be delivered through our concentric tube robots designed for beating-heart surgery in the right atrium. The design requirement is based on the innermost robot tube that has a $1.6 \mathrm{~mm}$ ID (sized to fit the $1.5 \mathrm{~mm}$ diameter of the device) and a radius of curvature of $25 \mathrm{~mm}$. The main components affected by this requirement are the rail and rod (Figure 7). To ensure sufficient strength of the rail's ratchet teeth and retaining rings, it was designed to bend with respect to the cross section axis parallel to the ratchet teeth. A finite element method (FEM) analysis was used to ensure that stresses were significantly less than the yield stress of $\mathrm{NiCo}$ $(\sim 900 \mathrm{MPa})$.

As described in the previous section on device removal, the rod prevents the distal wings from folding outward during normal operation. This means that when the device is pressing tissue together between the distal and proximal wings, the rail is experiencing a tensile load while the rod is experiencing a compressive load. The tensile load in the rail is given by the desired pull-out force of $1 \mathrm{~N}$. Assuming that the pull-out force is applied to the midpoint of the distal wings, the 3:1 ratio of moment arms means that the rod must support a compressive column load of $3 \mathrm{~N}$. The rail and base components provide substantial bracing for the rod acting as a column. The assumption of hinged-hinged boundary conditions was used to conservatively select a rod diameter of $150 \mu \mathrm{m}$ to avoid failure by buckling.

Other parts that required FEM analysis to ensure a design of sufficient strength to meet the pull-out force requirement include the ratchet teeth, the ratchet pawl, the four revolute joints of the wings and the wings themselves (Figure 7).

\subsection{Flexure design}

While the device design uses traditional macro-scale mechanical components such as screws and revolute joints, it also utilizes flexure joints to accomplish sub-millimeter motion. For example, the ratchet pawl deflects $100 \mu \mathrm{m}$ as it slides over the ratchet teeth. Its design required that it be flexible enough to achieve this deflection without yielding while also being sufficiently stiff to prevent deflection-induced ratchet disengagement when the device is loaded to the desired pull-out force.

Flexure springs were also designed to force the wings to rotate open by $30^{\circ}$ as the device is extended from the robot tube (Figure 6). The springs are necessary to ensure that the wings do not remain stuck against the rail and rod of the device. It was determined that if they are initially spread by at least $30^{\circ}$ from the folded configuration then contact forces with the tissue during ratcheting would cause them to open fully. To achieve this angular rotation, the wing springs deflect by $160 \mu \mathrm{m}$ when the wings are folded. FEM analysis was used to size the springs such that Von Mises stress remained below the yield stress. 


\section{Robotic device delivery}

In contrast to standard robots possessing rigid links and discrete joints, concentric tube robots are a type of continuum robot. When their constituent pre-curved tubes are inserted inside each other, their common axis conforms to a mutual resultant curvature. By controlling relative translations and rotations of the tubes at their proximal ends, the shape and length of the robot can be varied. Thus, as depicted in the example of Figure 9, the tubes act as both links and flexure joints.

A design methodology for concentric tube robots has been proposed by Dupont et al. (2010) and Bedell et al. (2011) that is based on the following four design rules.

1. Telescoping dominant stiffness. The stiffnesses of the tubes are selected such that each telescoping section dominates all of those sections extending from it. The result is that the shape and displacement of each telescoping section is kinematically decoupled from that of the proximal sections.

2. Fixed and variable curvature sections. Each telescoping section is designed to have either fixed or variable curvature. A fixed curvature section relaxes to the shape of its pre-curvature when it is extended from the preceding section. In contrast, a variable curvature section can take on a continuous range of curvatures usually ranging between zero (straight) and a maximum value. A single tube is required to construct a fixed curvature section while two tubes are needed to construct a variable curvature section (Dupont et al. 2010). This design rule is analogous to defining the types of joints (and, thus, link motions) of the robot.

3. Piecewise constant initial tube curvatures. When using telescopic extension to navigate narrow curved passages or penetrate tissue, the only robot shape that does not produce lateral motion or forces is one of piecewise constant curvature. During telescopic extension, the order of extension must proceed from the proximal section to the distal one. It has been demonstrated that by pre-curving each tube such that its curvature is piecewise constant, the combined telescoping curvature is also approximately piecewise constant (Dupont et al. 2010).

4. Increasing curvature from base to tip. Larger section curvatures produce larger rotations and off-axis translations and for a given section length. By employing larger curvatures for the distal sections, these sections can be designed to possess a significantly sized workspace at the robot tip without relying on motion of the proximal sections.

In summary, tube diameters and initial curvatures are selected such that the robot behaves as a concatenation of kinematically independent fixed curvature and variable curvature sections. Referring back to Figure 9, each fixed curvature section has two kinematic input variables, $\{1, \theta\}$ and contributes two degrees of freedom corresponding to the extension length and rotation of the section. Since variable curvature sections are constructed from two tubes, they possess three kinematic input variables $\left\{\theta_{1}, \theta_{2}, l\right\}$ and contribute three degrees of freedom to the robot. The angles $\left\{\theta_{1}, \theta_{2}\right\}$ control rotation and curvature of the section and $I$ controls arc length.

\subsection{Robot design}

Using these design rules, the robot design problem is to select (i) the number of telescoping sections, (ii) the type of each section (fixed or variable curvature) and (iii) the curvature and maximum length of each section based on surgical-task workspace requirements and anatomical constraints. Algorithms for numerically computing these parameters have been presented by Bedell et al. (2011) and Anor et al. (2011). 
In particular, Bedell et al. (2011) decompose the robot and the design problem into two parts in which the proximal portion of the robot is responsible for navigation to the surgical site through telescopic extension and the distal portion of the robot is responsible for manipulating tools and tissue at the surgical site. This decomposition is depicted in Figure 10 where the robot navigation sections of the robot extend from percutaneous entry into the internal jugular vein to entry into the right atrium. Once positioned, these sections remain fixed during the surgery. The distal manipulation sections, as shown in Figure 10(b), extend from navigation sections and are responsible for manipulating the septum secundum and primum as well as device deployment.

Additional design constraints arise from mechanical considerations of the tubes, e.g. maximum strain, and from the task. In this case, the task consists of delivering a $1.5 \mathrm{~mm}$ approximation device through the lumen of the robot. To satisfy this constraint, the inner diameter of the innermost tube was specified as $1.6 \mathrm{~mm}$.

Following the approach of Bedell et al. (2011), the manipulation workspace was defined on the septum and an iterative process was used to arrive at a robot design that simultaneously satisfied anatomical, task, tool and mechanical constraints. As depicted in Figure 11 and detailed in Table 1, the resulting design possesses seven degrees of freedom and is comprised of three telescoping sections. Note that the table lists the radius of curvature (reciprocal of curvature) of each section and that infinite radius of curvature corresponds to the section being straight.

The proximal section is of fixed curvature and is used to navigate from the point of percutaneous entry to the internal jugular into the right atrium. The distal sections of the robot are of variable and fixed curvature, respectively, and are the sections responsible for positioning and deploying the approximation device. A $0.778 \mathrm{~mm}$ clearance is used between sections 1 and 2 to accommodate an echogenic PVDF coating of thickness $0.25 \mathrm{~mm}$ on section 2, which is designed to eliminate ultrasound imaging artifacts from this section.

\subsection{Drive system}

The drive system depicted in Figure 12 enables actuation of robot designs comprised of up to three actively controlled sections. System modularity allows the section drives to be swapped in and out to achieve any desired arrangement of variable and fixed curvature sections. Each section drive consists of a linear stage with either one or two rotary stages for fixed and variable curvature sections, respectively.

The nose cone shown in Figure 12 serves two purposes. First, it prevents interference between the drive system body and the head of the patient when the robot is positioned to enter the patient's neck. In addition, it enables the inclusion of multiple passive, manually controlled robot sections in addition to the three actively controlled sections. These passive sections are typically used as proximal navigation sections. For the specific design used here and as shown in Figure 11, section 1 is a passive section while sections 2 and 3 are actively controlled.

As shown in Figure 13, the robot is designed so that the lumen of the distal tube extends from the robot tip back to the drive system. This provides a continuous channel for deploying a sequence of tools and devices at the robot's tip without removing it from the body.

For procedures inside the beating heart, it is necessary to prevent blood from leaking out through the robot lumen and also to prevent air from leaking into the heart. This is accomplished by means of a system of silicone diaphragm seals and flush ports. Prior to 
insertion into the body, all sealed chambers are flushed with heparinized saline to remove air and minimize the formation of emboli.

The first seal is incorporated into the proximal end of the lumen as labeled in Figure 13. The approximation device is protected during insertion through the seal by an enclosing sheath as depicted in the inset. Once through the seal, it slides through a tube of constant diameter to reach the distal end of the robot.

The clearance between the tubes of sections 2 and 3 are sufficiently small that no sealing between the three tubes comprising these sections is necessary. A seal is included, however, where the tubes of section 2 enter the fixed proximal end of the tube comprising section 1 , owing to the larger clearance. The white seal cover and flush tube can be seen in Figure 12 just behind the tip of the nose cone.

\subsection{Robot control}

Figure 14 depicts the block diagram of the robot controller. There are two input modes of teleoperated control. In the first, a Phantom Omni haptic interface is used to control robot tip position and orientation. Alternately, a graphical user interface can be used in combination with a keyboard to produce individual and collective motions of the tubes. During initial in vivo tests, both modes were evaluated. Since the cardiac surgeon preferred to operate the ultrasound imaging system himself and this often required two hands, it was determined to be most efficient for the surgeon to orally provide commands in which relative robot motions were specified in terms of millimeters, degrees and directions. These could be most accurately carried out by a second person through keyboard input and so this approach was adopted.

The kinematic model used in the controller is detailed in Dupont et al. (2010). It assumes that each tube can bend and twist and that the tubes can possess arbitrary initial curvature and stiffness. Effects that are neglected include shear of the cross section, axial elongation, nonlinear constitutive behavior, friction between the tubes (Lock and Dupont, 2011) and deformation due to external loading (Lock et al., 2010; Rucker et al., 2010b; Mahvash and Dupont, 2011). While the latter effects are not necessarily negligible, image-guidance during teleoperation enables the robot to be easily guided to the desired location.

The forward kinematic model consists of a non-linear second-order differential equation with split boundary conditions that describes robot curvature as a function of arc length. To compute the tip configuration, curvature must be integrated along the length of the robot. To enable real-time computation of the forward and inverse kinematics, the forward model is pre-computed over the robot's workspace and accurately approximated by a product of truncated Fourier series (Dupont et al., 2010). The inverse kinematic solution is solved at each time step using a root finding method applied to the functional approximation that typically converges in five or fewer iterations. Our current unoptimized implementation can compute up to eight iterations during the $1 \mathrm{~ms}$ time step of our $1 \mathrm{kHz}$ controller.

The controller is implemented in Windows using a soft real-time implementation. The process includes two time-critical user mode threads running at $1 \mathrm{kHz}$ that implement the controllers and an application thread that updates a GUI. One of the time-critical threads executes the PID controller of the robot arm and the other executes the inverse kinematics block. 


\section{Experiments}

To evaluate the device, three types of experiments were conducted. First, the mechanical strength of the tissue approximation device was measured by testing three devices to failure. Next, the surgical procedure was developed through ex vivo experiments performed on the bench top. Finally, a set of in vivo PFO closures were performed in a porcine model. Each set of experiments is described below.

\subsection{Pull-out force testing}

Owing to the modest pressure difference between the right and left atria, an approximation device experiences much less tensile load than the design specification of $1 \mathrm{~N}$. Nevertheless, experiments were performed to evaluate the strength of the approximation device.

Such a repair can fail in one of two ways. The approximated tissue may tear or the device may fail. In the case of a surgical repair, the septal tissue will always tear before the suture fails. Consequently, it is important to avoid applying a force much larger than the desired 1 $\mathrm{N}$ approximation force. The device wingspan of $6 \mathrm{~mm}$ and wing width of $1 \mathrm{~mm}$ are comparable to the width of a suture bite and the width of the suture, respectively. Thus, the device strength should exceed the desired $1 \mathrm{~N}$ approximation force by a safety factor.

Three devices were tested to failure as shown in Figure 15. A tensile testing machine was used with a test fixture consisting of parallel metal plates with $1.5 \mathrm{~mm}$ diameter holes through which the rail and rod of Figure 7 passed. As shown, all devices failed at loads above $10 \mathrm{~N}$ providing a safety factor of 10 . The failures all corresponded to over-rotation of the wings.

\subsection{Ex vivo procedure development}

To develop the detailed procedural workflow for robotic deployment of the approximation device, ex vivo experiments were performed using porcine hearts obtained from the supermarket. These experiments simulated PFO closure although no PFO was present or created during the experiments. As shown in Figure 16, a fixture was constructed to support the heart in the desired surgical orientation. The robot was deployed into the right atrium through the superior vena cava. A portion of the right atrial free wall was removed to enable visual observation of the atrial anatomy during the procedure.

In the close-up view of the anatomy in Figure 17, the entrance to the PFO channel is visible. Dashed lines indicate its direction between the two tissue layers of the septum secundum and septum primum. It can be seen that the ridge of septum secundum running over the entrance to the PFO channel is composed of thick tissue. The septum primum tissue that lies underneath this ridge is either quite thin or is missing. Thus, it is necessary to stretch the ridge tissue over the entrance of the channel far enough that it can be approximated with sufficiently thick tissue of the septum primum to avoid tear-out during approximation. (See the direction of stretching shown with arrows in Figure 1(b).)

This is accomplished by using a stylet extended from the robot tip to penetrate into the ridge tissue. Using the penetration to grip the tissue, the robot stretches the ridge tissue over the septum primum as shown in Figure 18. The stylet and robot tip are then driven completely through both tissue layers into the left atrium.

At this point, the stylet can be removed while the robot is held in position to maintain the desired location of the approximation device. The device is now loaded into the proximal end of the robot for deployment and is deployed following the sequence of steps shown in Figure 6. The resulting sealed channel is shown in Figure 19. 
The steps of the PFO closure procedure are enumerated in Table 2 . The procedure comprises two major parts: (1) robot navigation and tissue manipulation and (2) approximation device deployment. While each was performed on the bench top using optical visualization, this is not possible inside the blood-filled beating heart. In the operating room, two imaging modalities are readily available for intracardiac surgery. These are ultrasound and fluoroscopy.

Using 3D ultrasound is preferable since it does not involve ionizing radiation and it can be used to visualize both the robot and the soft tissue. Its resolution is limited to $1-2 \mathrm{~mm}$, however, and imaging artifacts arise in the vicinity of the robot tip. While it does not display soft tissue, fluoroscopy is useful as a complementary imaging modality since its submillimeter resolution enables detailed visualization of the approximation device. These relative benefits led to the imaging strategy of Table 2 in which ultrasound was used for guiding robot motions and fluoroscopy was used to guide device deployment. This approach proved successful for the in vivo trials described in the next section.

\subsection{In vivo testing}

To validate the procedure developed during ex vivo testing, three in vivo trials were performed using 50-70 kg Yorkshire pigs. This species was selected owing to the similarity between its heart and the human heart (Suematsu et al., 2005). In particular, septal motion during the cardiac cycle is very similar. These procedures lasted about 2 hours, which is much shorter than many catheter interventions.

As shown in Figure 20, the robot enters the pig percutaneously through the right internal jugular vein. While in a human, transesophageal echo can be employed to visualize the atrial septum, this is not possible in our animal model since the lungs lie between the esophagus and the heart. Consequently, the chest is opened as shown to enable placement of the ultrasound probe (shown in the top of the image) directly on the atrial free wall.

Insertion of the robot into the vasculature proceeds in several steps to avoid damage to the vessel walls. At each step, every cannula and tube is flushed with heparinized saline. First, a guide wire and dilator are sequentially inserted into the right atrium. A $16 \mathrm{Fr}$ plastic cannula with a seal and flush port is then inserted over the dilator and the dilator and guide wire are removed. The cannula length matches the length of the navigation portion of the robot (Figure 10(a)). For the duration of the surgery, the cannula is held fixed in the vasculature while the robot slides back and forth inside it.

Next, the surgeon manually inserts the navigation tube of the robot through the cannula and into the right atrium. With this tube in position, the support mechanism of the robot drive system (not shown) is adjusted so that the navigation tube can be locked into the nose cone of the drive system. The manipulation sections of the robot are then driven forward into the navigation tube and the steps of Table 2 are followed.

Examples of intraoperative images are shown in Figure 21. Real-time 3D ultrasound (Philips IE 33) provides image volumes updated at $30-35 \mathrm{~Hz}$ as shown in Figure 21(a). While the image is noisy, several factors aid in image interpretation. Heart motion during real-time imaging facilitates distinguishing the robot from the tissue. Furthermore, while it is difficult in the figure to distinguish the robot tip from the septal tissue as the robot approaches contact, changes in tissue motion aid in understanding how hard the robot is pressing against the tissue. For example, to detect contact, the surgeon would observe the ultrasound volume from multiple viewpoints and look for changes in tissue motion over the cardiac cycle. The ability of the robot to hold a fixed configuration during this imaging was crucial to successfully monitoring tissue interaction. 
Fluoroscopic imaging was used for visualization during device deployment as described in Table 2. While the tissue is not visible, fluoroscopy provided the benefit of being able to see the approximation device inside the robot. During deployment, forces between the device and tissue were inferred from motions of the device. For example, each set of wings would rotate fully open when loaded against tissue. Using these cues, it was possible to monitor the deployment process in the absence of force feedback.

Three animal trials were performed and in each of the cases the septum secundum and primum were successfully approximated. By coincidence, one of the three animals had a naturally occurring $9 \mathrm{~mm}$ wide PFO and we were able to verify using ultrasound that the approximation device sealed it. For the other two cases, the PFO channel was not patent, but we were still able to demonstrate successful device placement and closure of the entrance to the channel.

For the animal with a naturally occurring PFO, leakage from the left to right atrium through the PFO can be observed under conditions of elevated left atrial pressure, which was achieved by forced lung ventilation. This was documented using color Doppler ultrasound imaging as shown in Figure 22(a). After placement of the approximation device, the flow was eliminated as shown in Figure 22(b). Postmortem views of the device inside the heart are shown from the right and left atrium in Figure 23.

\section{Discussion}

The concept of adapting procedures and tooling to the capabilities of the robot is what made robots successful in manufacturing. The approach can be applied equally effectively to medical applications. This approach has been applied here to the use of concentric tube robots for a specific intracardiac procedure through the design of a tissue approximation device for PFO closure.

The device was manufactured using a metal MEMS process that enables the fully assembled fabrication of macroscale mechanisms in millimeter-scale devices. This has the effect of reducing the complexity of the robot motions required to deploy it. When deployed, the wings of the device are flush with the tissue surface and thus will be quickly encapsulated in tissue. This is especially important on the left atrial side to avoid the formation of emboli that could travel to the brain or the heart. The comparison with an existing occlusion device in Figure 2 clearly demonstrates the superiority of the proposed approach in terms of the amount of foreign material exposed to the blood flow. On the right side of the heart, the protruding ratchet rail shown in Figure 23(a) is comparable in size to the suture knots of a surgical repair as shown in Figure 1(c) and so will not be problematic.

Successful beating-heart PFO closure using this device was made possible by the use of a concentric tube robot as the delivery platform. The robot was able to enter the heart percutaneously and provide millimeter-scale positioning accuracy within the right atrium, which was required to ensure adequate targeting of the ridge above the PFO channel as shown in Figure 17. Ultrasound image quality was the limiting factor in positioning.

Since the bending stiffness of the robot exceeds that of catheters by several orders of magnitude, the robot can manipulate tissue with greater stability and precision. If the same procedure were attempted with a catheter, it is likely that the catheter could be positioned with sufficient accuracy with respect to the septum secundum. Its compliance, however, would make it extremely difficult to accurately stretch this tissue to overlap the septum primum. In addition, if a PFO was large enough to require several devices for closure, obtaining the necessary relative positioning of the devices would also likely be difficult. 
A substantial advantage of the robot is its ability to hold its position during imaging. During the surgery, the surgeon would pause regularly to reassess the relative configuration between the robot, the tissue and the approximation device. During these pauses, there was no risk that the robot would change position inside the heart. This held true even when the tip of the robot pressed against the septal tissue. Furthermore, no adverse effects from having the robot inside the heart, e.g. arrhythmias, were observed.

The combination of concentric tube robots and novel millimeter-scale tooling has broad potential in converting open-heart procedures to beating-heart interventions. Example applications include tissue removal to correct outflow tract obstructions as well as mitral valve repair. Since these portions of the heart experience greater motion during the cardiac cycle than the atrial septum, it is likely that enhanced sensing and control techniques (e.g. Mahvash and Dupont, 2011), will be needed for these applications. The technology is also applicable to minimally invasive surgery inside other body lumens including neurosurgical and urological procedures.

\section{Acknowledgments}

This paper was presented in part at the 2011 IEEE International Conference on Robotics and Automation.

Funding

This work was supported by the National Institutes of Health (grant numbers R01HL073647 and R01HL087797).

\section{References}

Anor T, Madsen J, Dupont PE. Algorithms for design of continuum robots using the concentric tubes approach: a neurosurgical example. Proceedings IEEE International Conference on Robotics and Automation. 2011:667-673.

Butler E, Folk C, Cohen A, et al. Metal MEMS tools for beating-heart tissue approximation. Proceedings IEEE International Conference on Robotics and Automation. 2011:411-416.

Bedell C, Lock J, Gosline A, Dupont PE. Design optimization of concentric tube robots based on task and anatomical constraints. Proceedings IEEE International Conference on Robotics and Automation. 2011:398-403.

Calvert PA, Rana BS, Kydd AC, Shapiro LM. Patent foramen ovale: anatomy, outcomes and closure. Nat Rev Cardiol. 2011; 8:148-160. [PubMed: 21283148]

Camarillo D, Carlson C, Salisbury K. Configuration tracking for continuum manipulators with coupled tendon drive. IEEE Transactions on Robotics. 2009; 25:798-808.

Camarillo D, Milne C, Carlson C, Zinn M, Salisbury JK. Mechanics modeling of tendon-driven continuum manipulators. IEEE Transactions on Robotics. 2008; 24(6):1262-1273.

Dearani JA, Ugurlu BS, Danielson GK, et al. Surgical patent foramen ovale closure for prevention of paradoxical embolism related cerebrovascular ischemic events. Circulation. 1999; 100(Suppl. II):II-171-II-175. [PubMed: 10567299]

Dupont PE, Lock J, Itkowitz B, Butler E. Design and Control of Concentric Tube Robots. IEEE Transactions on Robotics. 2010; 26:209-225. [PubMed: 21258648]

Ikeuchi M, Ikuta K. Development of pressure-driven micro active catheter using membrane micro emboss following excimer laser ablation (MeME-X) process. Proceedings IEEE International Conference on Robotics and Automation. 2009:4358-4361.

Jayender J, Patel RV, Nikumb S. Robot-assisted active catheter insertion: algorithms and experiments. The International Journal of Robotics Research. 2009; 28:1101-1117.

Kerut EK, Norfleet WT, Plotnick GD, Giles TD. Patent foramen ovale: a review of associated conditions and the impact of physiological size. Journal of the American College of Cardiology. 2001; 38:613-623. [PubMed: 11527606] 
Khairy P, O'Donnell CP, Landzberg MJ. Transcatheter closure versus medical therapy of patent foramen ovale and presumed paradoxical thromboemboli: a systematic review. Annals of Internal Medicine. 2003; 139:753-760. [PubMed: 14597460]

Kizer JR, Devereux RB. Patent foramen ovale in young adults with unexplained stroke. New England Journal of Medicine. 2005; 353:2361-2372. [PubMed: 16319385]

Lock J, Dupont PE. Friction modeling in concentric tube robots. Proceedings IEEE International Conference on Robotics and Automation. 2011:1139-1146.

Lock J, Laing G, Mahvash M, Dupont PE. Quasistatic modeling of concentric tube robots with external loads. Proceedings IEEE/RSJ International Conference on Intelligent Robots and Systems (IROS). 2010:2325-2332.

Madhanir A, Niemeyer G, Salisbury JK. The black falcon: a teleoperated surgical instrument for minimally invasive surgery. Proceedings IEEE/RSJ International Conference on Intelligent Robots and Systems. 1998:936-944.

Mahvash M, Dupont PE. Stiffness control of continuum surgical manipulators. IEEE Transactions on Robotics. 2011; 27:334-345.

Mareedu RK, Shah MS, Mesa JE, McCauley CS. Percutaneous closure of patent foramen ovale: a case series and literature review. Clinical Medical Research. 2007; 5:218-226.

Meeker DC, Maslen EH, Ritter RC, Creighton FM. Optimal realization of arbitrary forces in a magnetic stereotaxis system. IEEE Transactions on Magnetics. 1996; 32:320-328.

Rucker D, Webster R III, Chirikjian G, Cowan N. Equilibrium conformations of concentric-tube continuum robots. The International Journal of Robotics Research. 2010a; 29:1263-1280.

Rucker DC, Jones BA, Webster RJ III. A geometrically exact model for externally loaded concentrictube continuum robots. IEEE Transactions on Robotics. 2010b; 26:769-780. [PubMed: 21566688]

Simaan N, Xu K, Kapoor A, et al. A system for minimally invasive surgery in the throat and upper airways. The International Journal of Robotics Research. 2009; 28:1134-1153. [PubMed: 20160881]

Suematsu Y, Martinez JF, Wolf BK, et al. Three-dimensional echo-guided beating heart surgery without cardiopulmonary bypass: atrial septal defect closure in a swine model. Journal of Thoracic and Cardiovascular Surgery. 2005; 130:1348-1357. [PubMed: 16256788] 


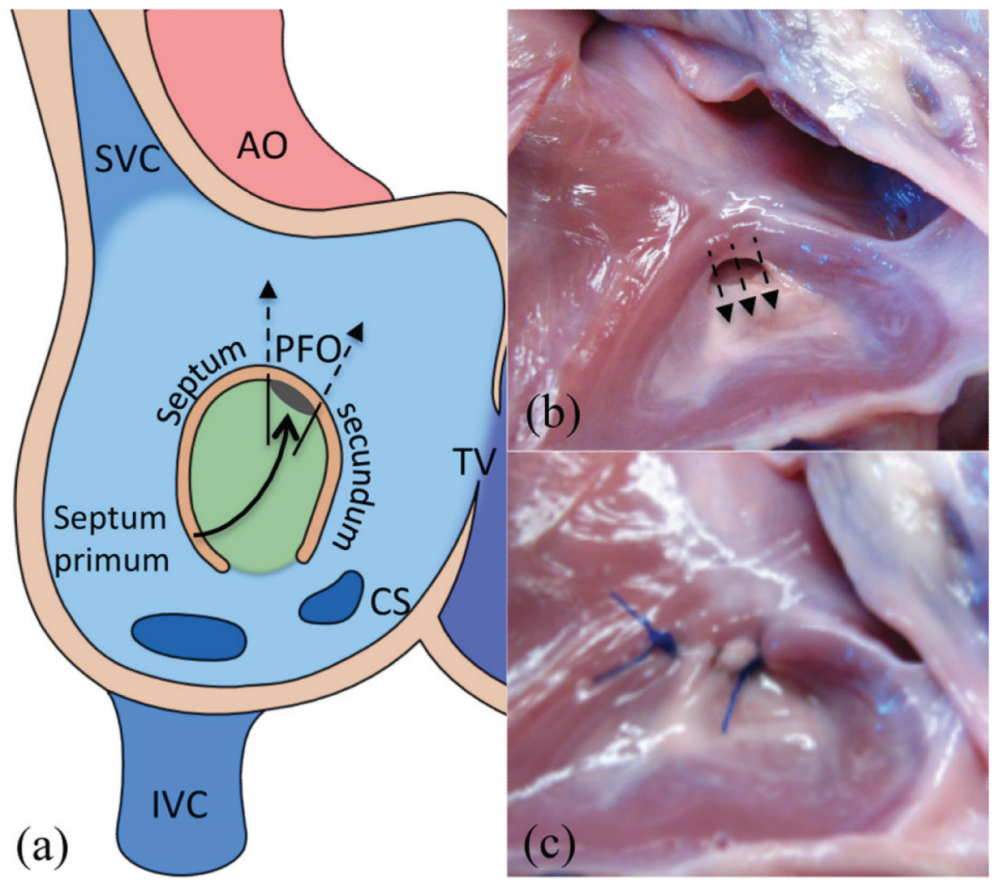

Fig. 1.

Patent foramen ovale (PFO) and surgical closure. (a) Right atrium view of PFO channel. (b) Right atrium view of porcine PFO. (c) Right atrium view of a surgically closed porcine PFO. SVC, superior vena cava; AO, aorta; CS, coronary sinus; TV, tricuspid valve; IVC, inferior vena cava. (Adapted by permission from Macmillan Publishers Ltd: Nature Review's Cardiology (Calvert et al., 2011), (C) 2011.) 


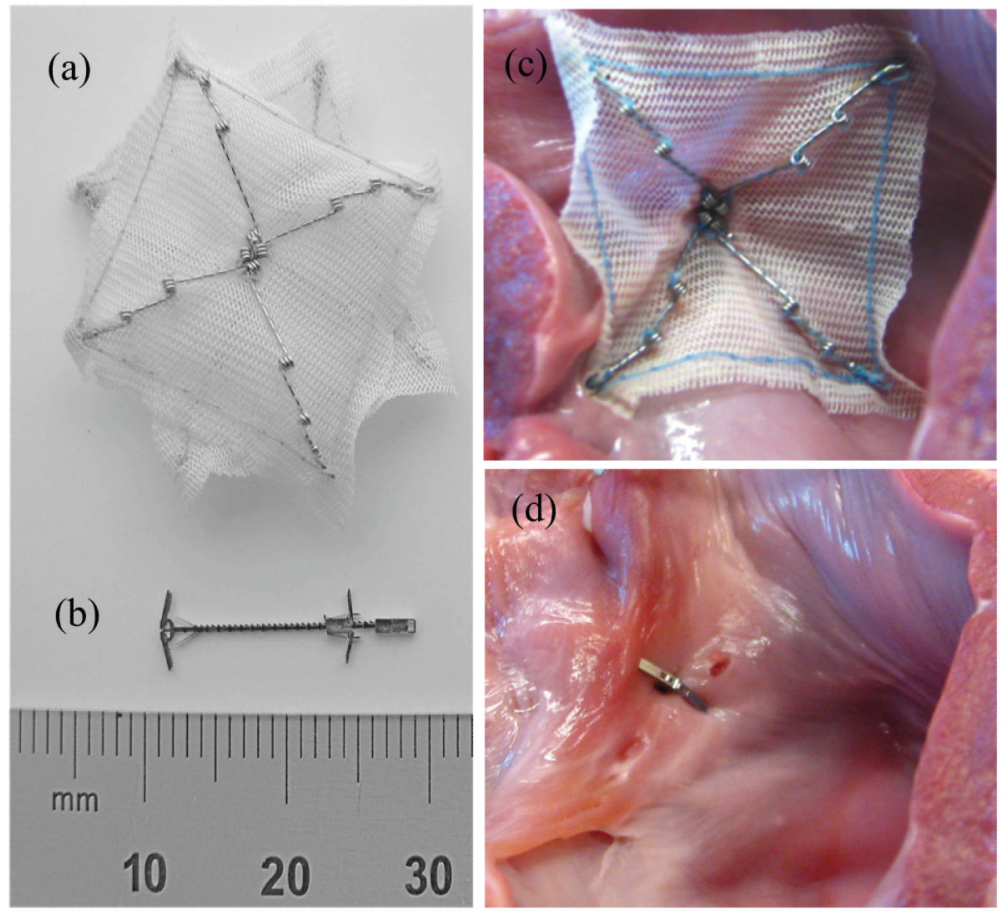

Fig. 2.

PFO closure devices. (a) CardioSeal catheter-delivered occlusion device, (b) Proposed metal MEMS tissue approximation device. (c) Left atrium view of the CardioSeal occluder in a porcine PFO. (d) Left atrium view of the proposed tissue approximation device sealing a porcine PFO. 


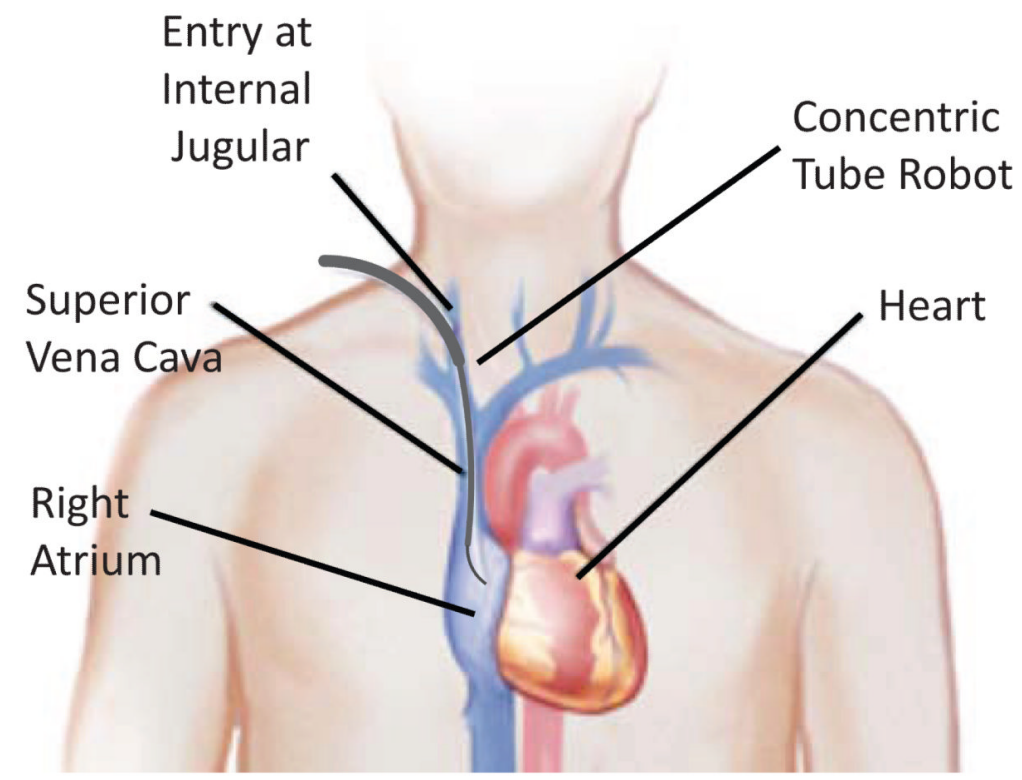

Fig. 3.

Concentric tube robot entering the beating heart via the internal jugular vein. 


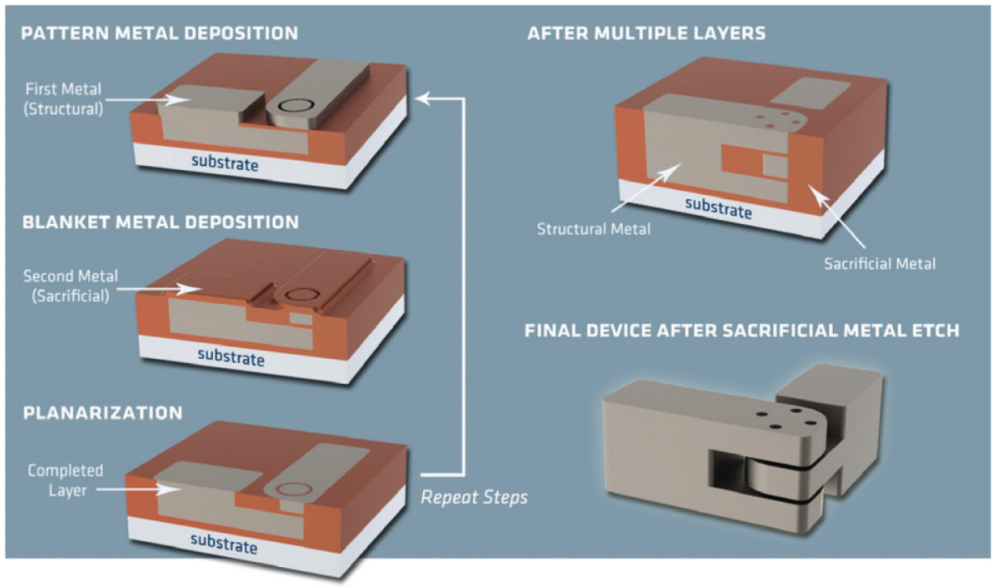

Fig. 4.

Metal MEMS fabrication process example for a revolute joint. The formation of each layer involves three steps: (a) pattern deposition of a sacrificial metal, (b) blanket deposition of structural metal, and (c) planarization. After all layers are formed, the sacrificial metal is removed, leaving behind the assembled $3 \mathrm{D}$ device. 


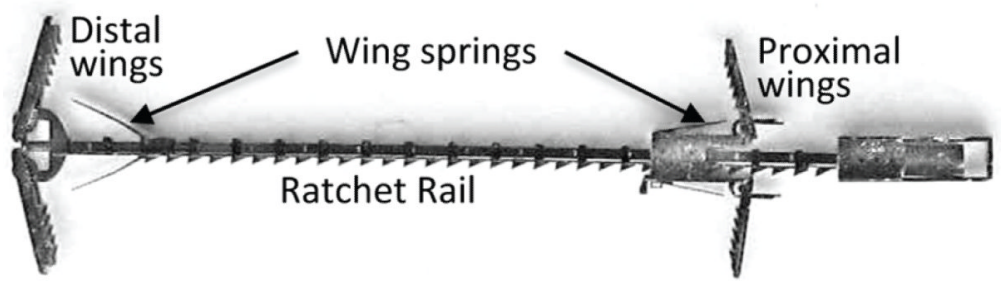

Fig. 5.

Metal MEMS tissue approximation device. 


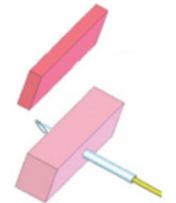

(1) Pierce $1^{\text {st }}$ laye
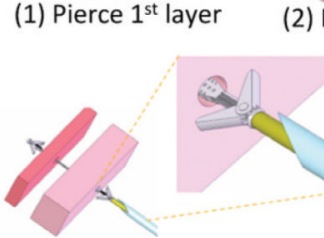

(4) Extend distal wings, then proximal wings

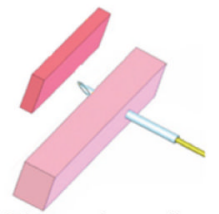

(2) Drag laterally

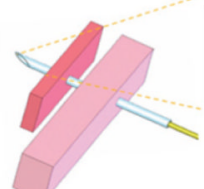

(3) Pierce $2^{\text {nd }}$ layer

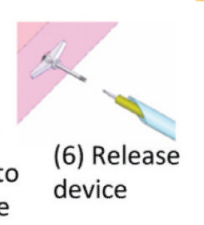

device

$1.5 \mathrm{~mm}$ folded

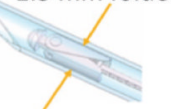

$6 \mathrm{~mm}$ wingspan

(5) Adjust ratchet to Approximate tissue
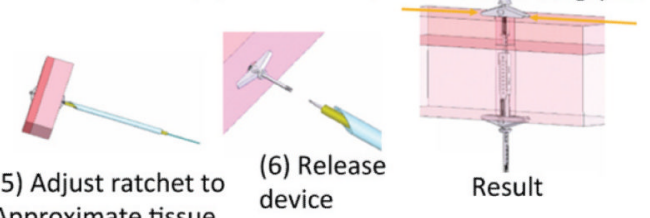

Fig. 6.

Tissue approximation device deployment sequence. 


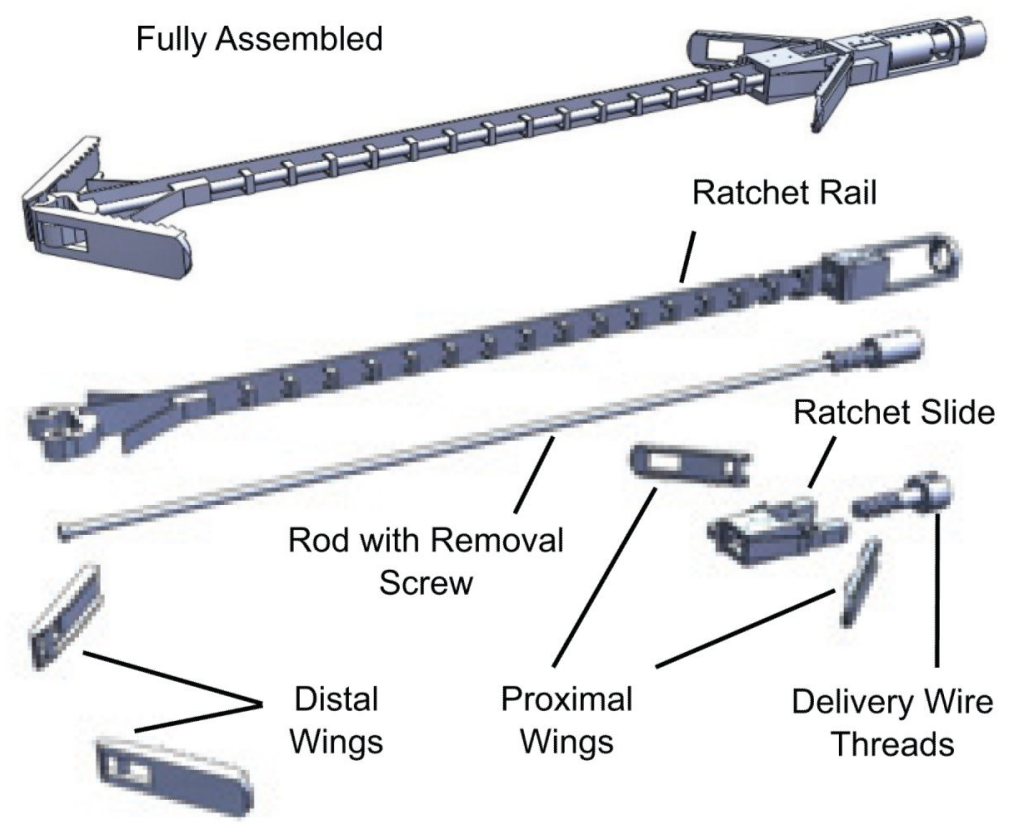

Fig. 7.

Tissue approximation device with expanded view of all components. 


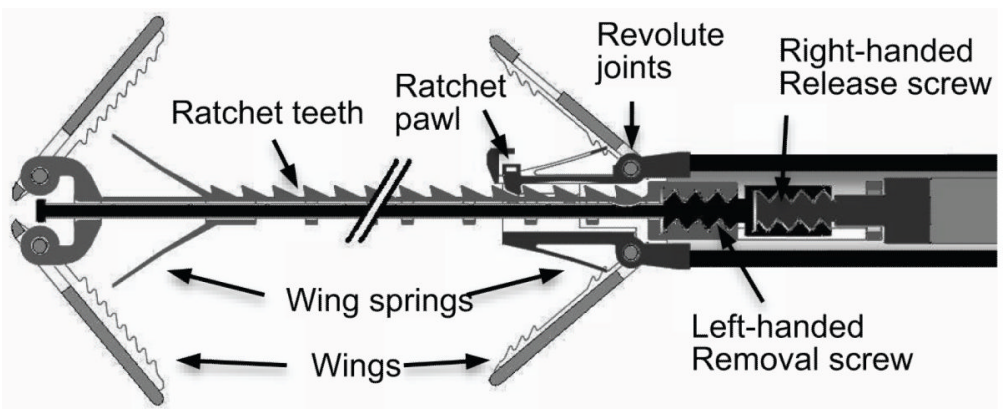

Fig. 8.

Screw mechanism concept for device removal by the release of distal wings. 


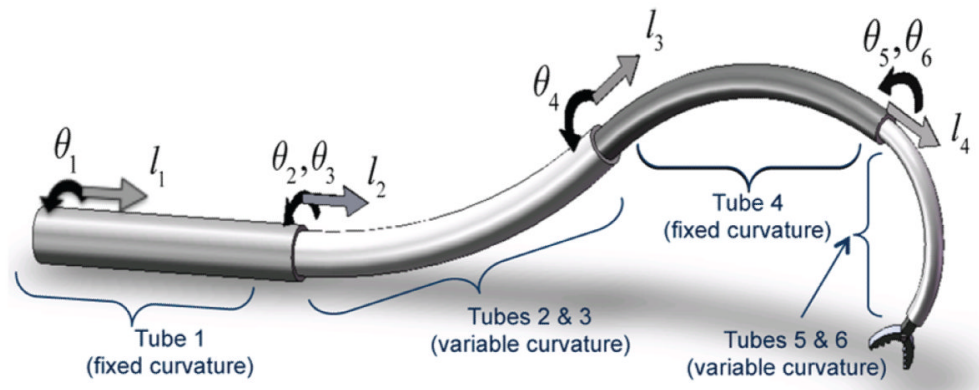

Fig. 9.

Conceptual architecture of concentric tube robot. Example shown is comprised of four telescoping sections that can be rotated and translated with respect to each other. 


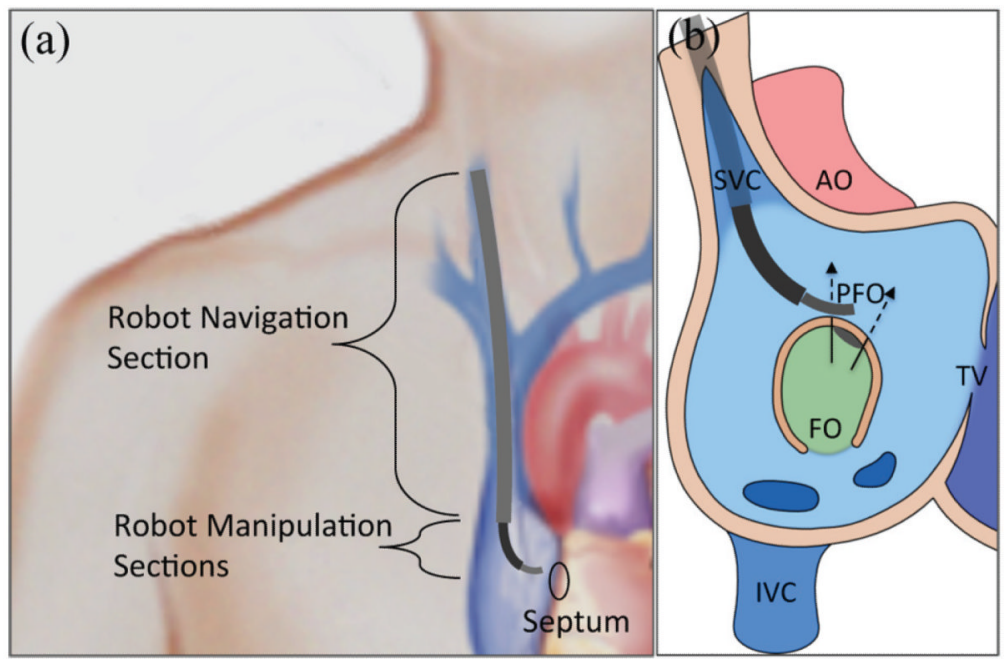

Fig. 10.

Decomposition of robot into navigation and manipulation sections: (a) overall view; (b) close-up of manipulation section inside right atrium. Adapted by permission from Macmillan Publishers Ltd: Nature Review's Cardiology (Calvert et al., 2011), (C 2011. 


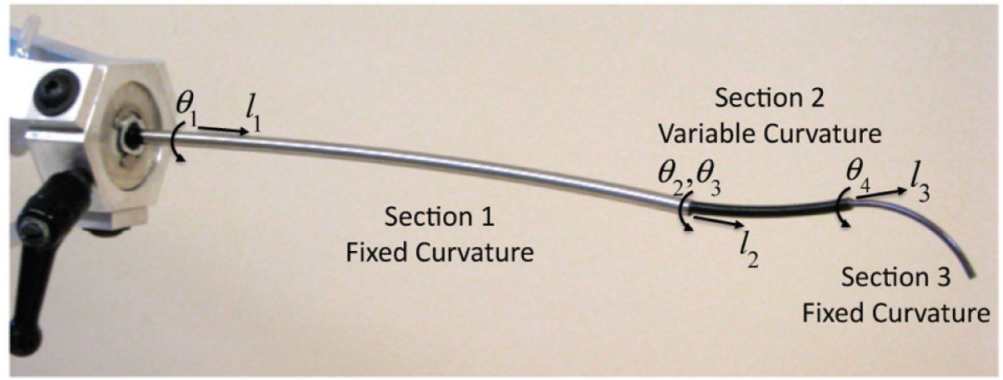

Fig. 11.

Robot used for PFO closure. The design consists of three telescoping sections as labeled. 


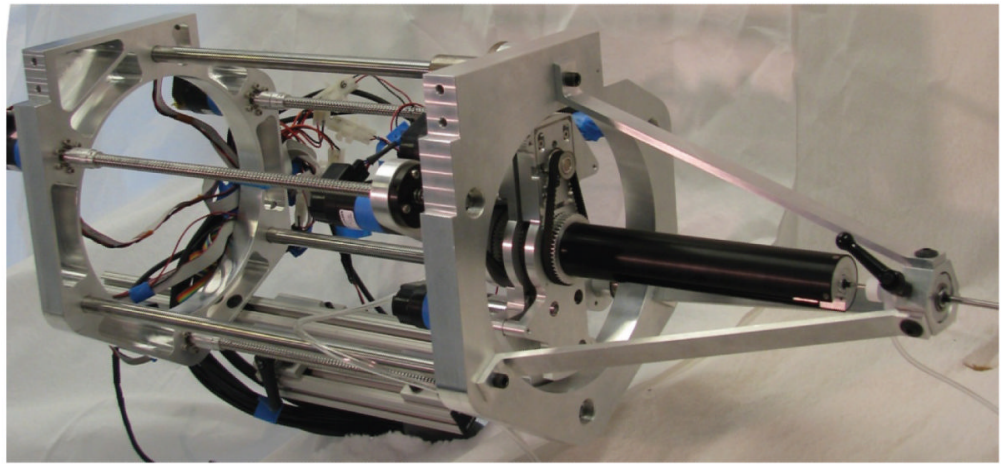

Fig. 12.

Robot drive system. 


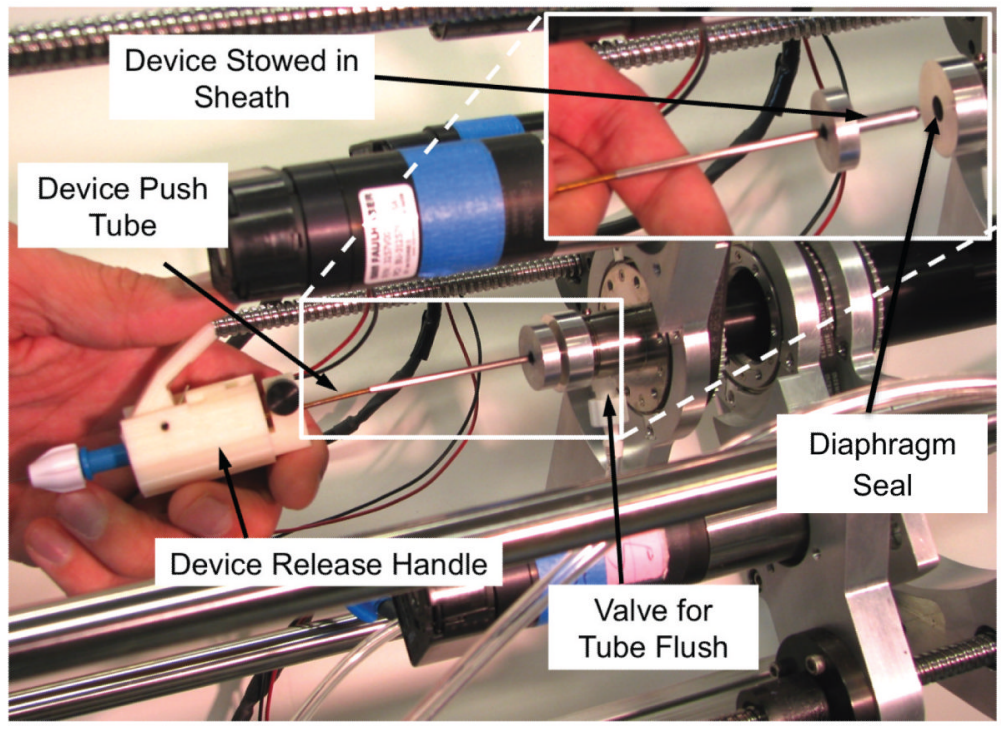

Fig. 13.

Rear-loading system for stylet and tissue approximation device. 


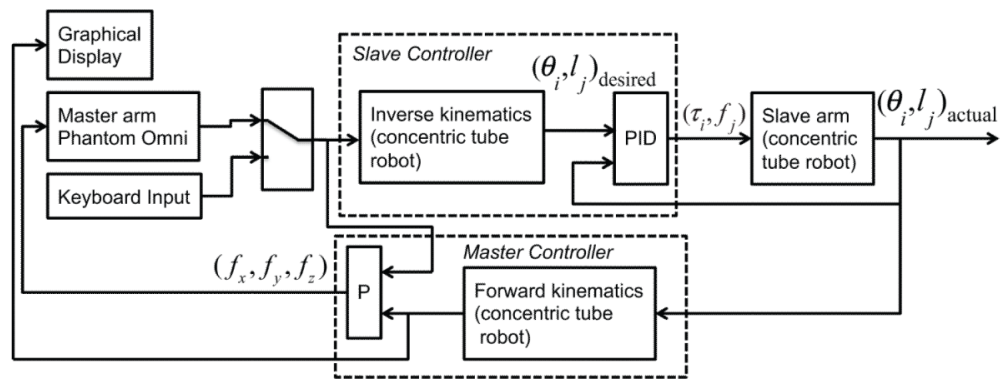

Fig. 14.

Control system block diagram. 


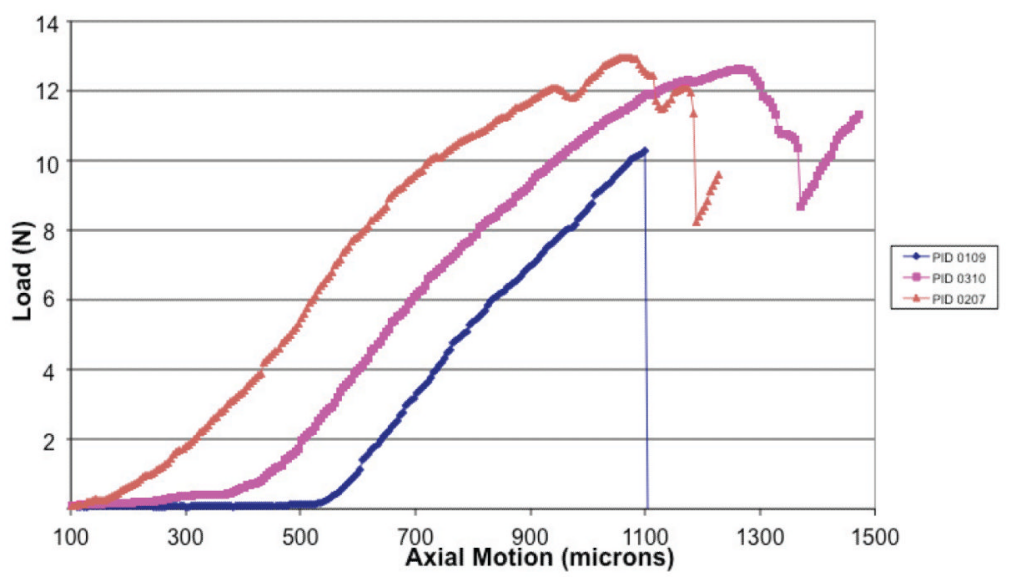

Fig. 15.

Device strength testing. Load versus displacement to failure. 


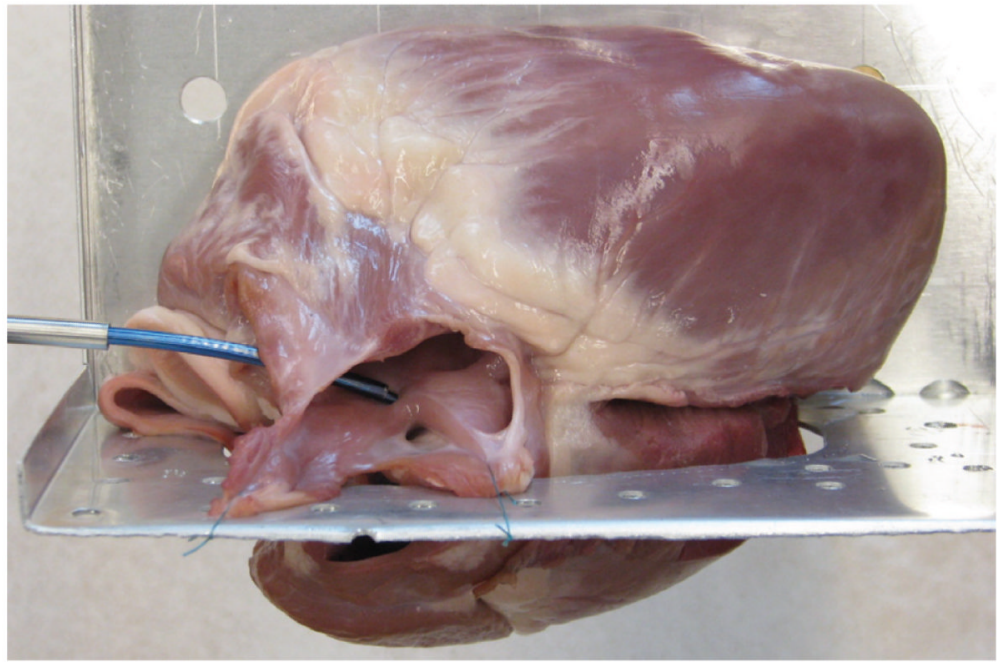

Fig. 16.

Porcine heart in a support fixture for ex vivo testing. Robot enters right atrium from the superior vena cava. 


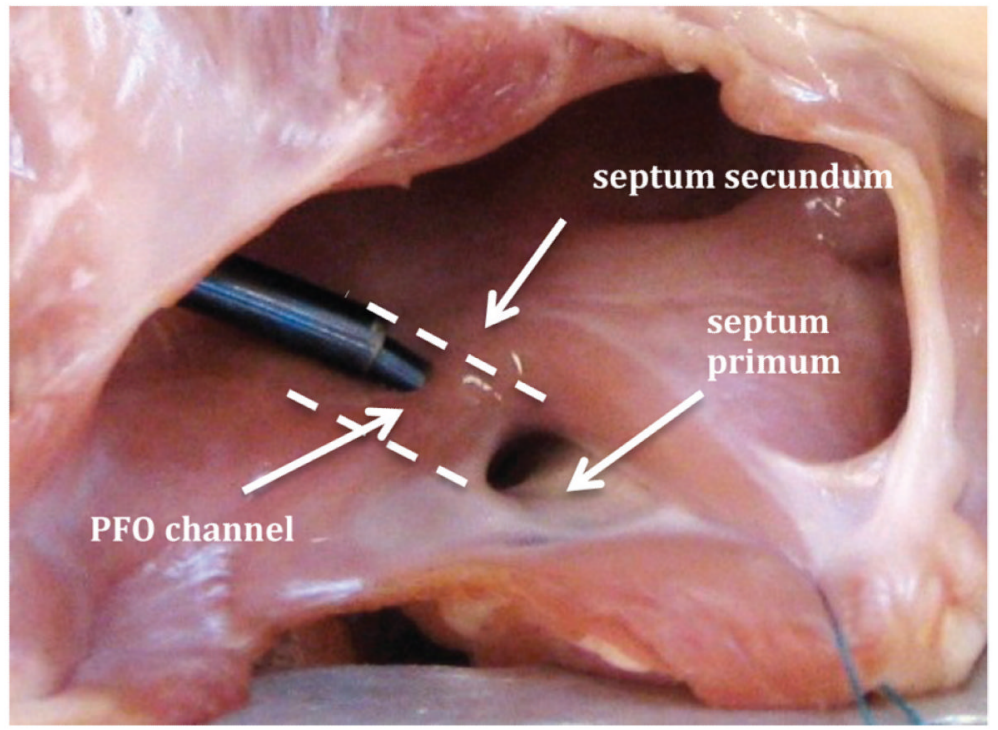

Fig. 17.

Cut-away view of right atrium during ex vivo porcine testing. Step 1: Robot is shown positioned on the ridge above the entrance to the PFO channel. Dashed lines show direction of channel between tissue layers. 


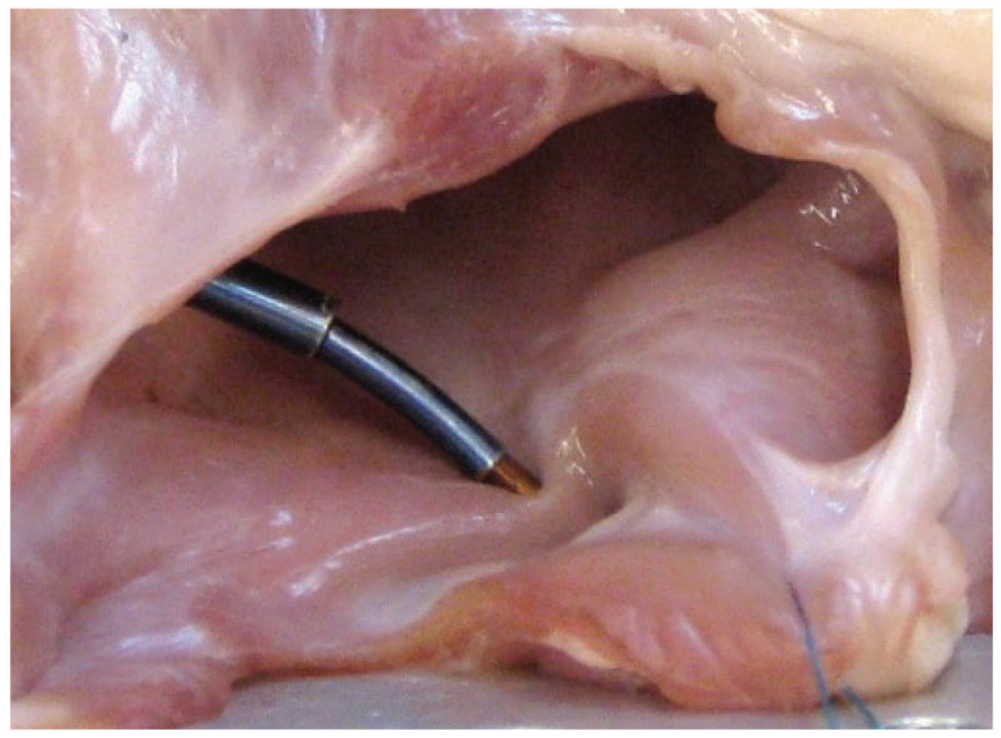

Fig. 18.

Step 3: Right atrium view showing the stylet extending into septum secundum ridge, which has been stretched over the opening to the PFO channel. 


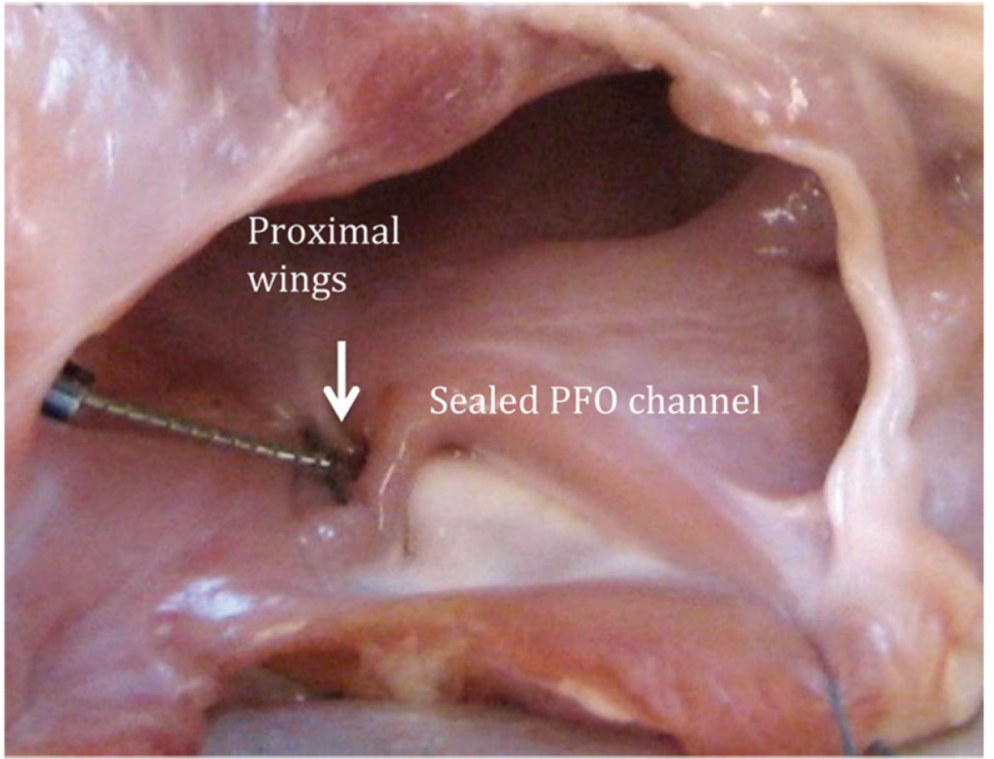

Fig. 19.

Step 14: Robot releasing the approximation device. 


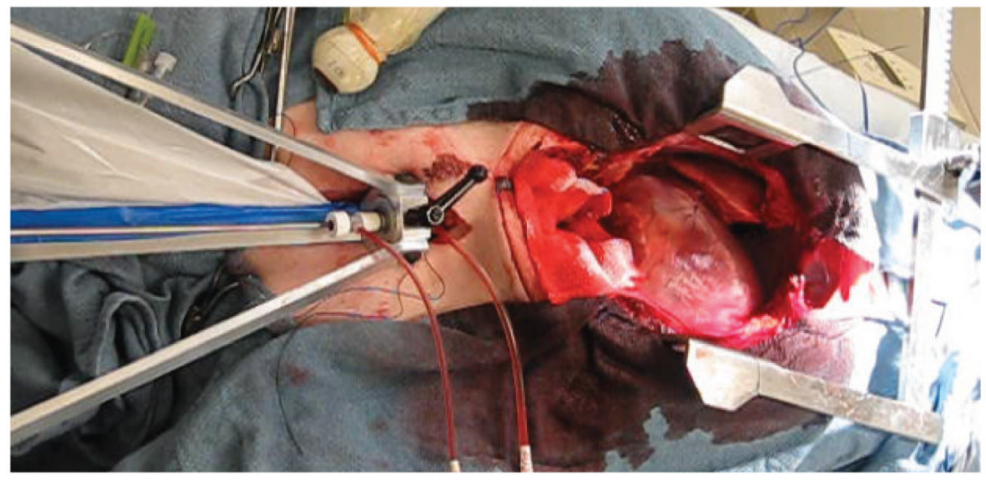

Fig. 20.

Open-chest model for ultrasound visualization. 

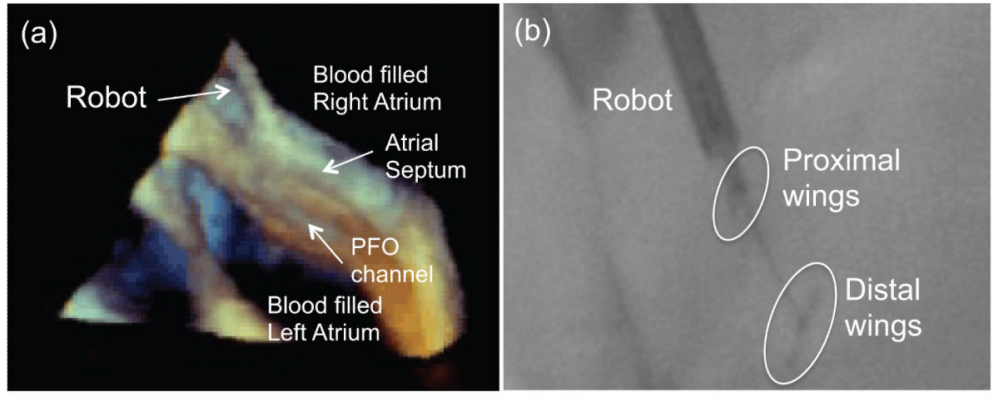

Fig. 21.

Surgical imaging: (a) robot navigation using 3D ultrasound; (b) device deployment using fluoroscopy. 

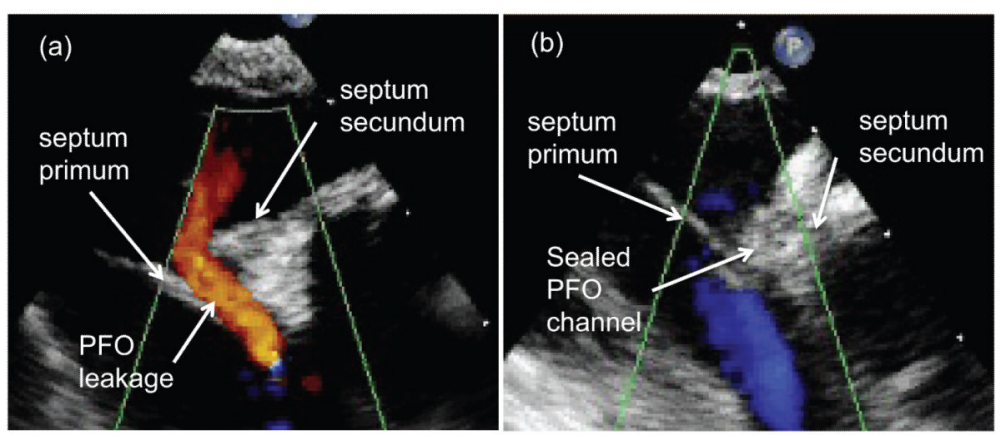

Fig. 22.

Color Doppler ultrasound images of PFO: (a) leakage from right to left atrium is visible before surgery; (b) leakage is eliminated after robotic closure. 

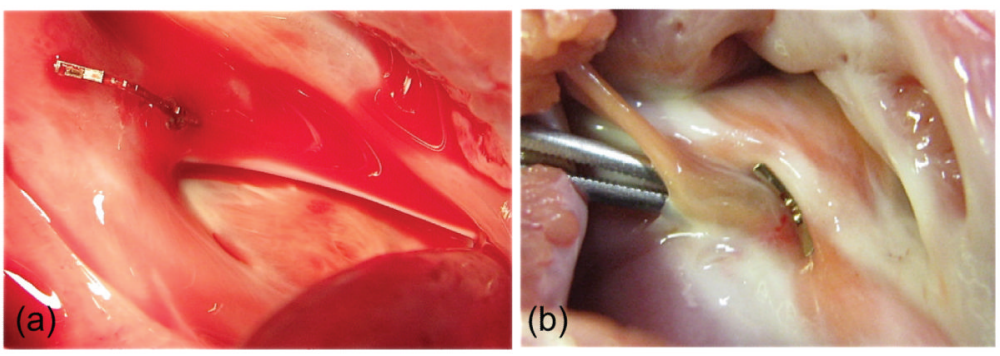

Fig. 23.

Implanted approximation device: (a) right atrial view; (b) left atrial view. 
Table 1

Robot Design parameters.

\begin{tabular}{lllll}
\hline & Navigation & \multicolumn{2}{l}{ Manipulation } \\
\cline { 3 - 4 } Section & $\mathbf{1}$ & $\mathbf{2}$ & $\mathbf{3}$ \\
\hline Curvature type & Fixed & Variable & Fixed \\
Material & Stainless steel & NiTi & NiTi \\
Maximum section length (mm) & 168 & 45 & & 35 \\
Radius of curvature (mm) & 700 & $80-\infty$ & & 25 \\
& & Outer Tube & Inner Tube \\
Inner diameter $(\mathrm{mm})$ & 3.42 & 2.438 & 1.943 & 1.600 \\
Outer diameter $(\mathrm{mm})$ & 4.18 & 2.642 & 2.311 & 1.829 \\
\hline
\end{tabular}


Table 2

\section{Surgical steps of robotic PFO closure.}

Using 3D real-time ultrasound for image guidance:

(1) Navigate to septum secundum and contact tissue ridge above PFO channel.

(2) Extend stylet from robot $(\sim 3 \mathrm{~mm})$ to partially penetrate septum secundum.

(3) Stretch tissue ridge of septum secundum so that it overlaps septum primum (direction of motion is as shown in Figure 1(b)

(4) Drive the stylet and robot through the entire thicknesses of the septum secundum and primum tissue layers and into the left atrium.

(5) Keeping the robot inserted through both tissue layers, remove the stylet through the proximal end of the robot.

(6) Insert the approximation device into the proximal end of the robot lumen as shown in the inset of Figure 13 and advance it through the lumen until it is just inside the distal tip.

Using fluoroscopic guidance:

(7) Deploy the distal wings of the approximation device from robot tip into left atrium.

(8) Holding the extended length of the approximation device constant, retract the robot tip into the right atrium.

(9) Retract the approximation device until the distal wings are fully deployed against the left atrial wall.

(10) Holding the extended length of the approximation device constant, retract the robot tip to expose the proximal wings of the device in the right atrium.

(11) Approximate the tissue by extending the device wire with respect to the device tube. This causes the device to ratchet such that the proximal wings move forward and press against the right atrial wall.

(12) Verify that the approximation device has deployed correctly.

Using 3D real-time ultrasound for image guidance:

(13) Verify with ultrasound that the device is properly positioned and with color Doppler that the PFO channel has been sealed.

(14) Release the device by rotating the device wire counterclockwise with respect to the device tube.

(15) Withdraw the robot. 